\title{
Activation of the Lectin Pathway of Complement in Pig-to-Human Xenotransplantation Models
}

\author{
Anjan K. Bongoni, ${ }^{1,2,3}$ David Kiermeir, ${ }^{3}$ Hansjörg Jenni, ${ }^{4}$ Annegret Wünsch, ${ }^{5}$ Andrea Bähr, ${ }^{5}$ \\ David Ayares, ${ }^{6}$ Jörg D. Seebach, ${ }^{7}$ Eckhard Wolf, ${ }^{5}$ Nikolai Klymiuk, ${ }^{5}$ Mihai A. Constantinescu, ${ }^{3}$ \\ Esther Vogelin, ${ }^{3}$ and Robert Rieben ${ }^{1,3,8}$
}

\begin{abstract}
Background. Natural IgM containing anti-Gal antibodies initiates classic pathway complement activation in xenotransplantation. However, in ischemia-reperfusion injury, IgM also induces lectin pathway activation. The present study was therefore focused on lectin pathway as well as interaction of IgM and mannose-binding lectin (MBL) in pig-to-human xenotransplantation models.

Methods. Activation of the different complement pathways was assessed by cell enzyme-linked immunosorbent assay using human serum on wild-type (WT) and $\alpha$-galactosyl transferase knockout (GalTKO)/hCD46-transgenic porcine aortic endothelial cells (PAEC). Colocalization of MBL/MASP2 with IgM, C3b/c, C4b/c, and C6 was investigated by immunofluorescence in vitro on PAEC and ex vivo in pig leg xenoperfusion with human blood. Influence of IgM on MBL binding to PAEC was tested using IgM depleted/repleted and anti-Gal immunoabsorbed serum.

Results. Activation of all the three complement pathways was observed in vitro as indicated by IgM, Clq, MBL, and factor Bb deposition on WT PAEC. MBL deposition colocalized with MASP2 (Manders' coefficient [3D] $\mathrm{r}^{2}=0.93$ ), $\mathrm{C} 3 \mathrm{~b} / \mathrm{c}\left(\mathrm{r}^{2}=0.84\right), \mathrm{C} 4 \mathrm{~b} / \mathrm{c}\left(\mathrm{r}^{2}=0.86\right)$, and C6 $\left(\mathrm{r}^{2}=0.80\right)$. IgM colocalized with MBL $\left(\mathrm{r}^{2}=0.87\right)$ and MASP2 $\left(\mathrm{r}^{2}=0.83\right)$. Human IgM led to dose-dependently increased deposition of MBL, C3b/c, and C6 on WT PAEC. Colocalization of MBL with IgM (Pearson's coefficient [2D] $\left.\mathrm{r}_{\mathrm{p}}{ }^{2}=0.88\right), \mathrm{C} 3 \mathrm{~b} / \mathrm{c}\left(\mathrm{r}_{\mathrm{p}}{ }^{2}=0.82\right), \mathrm{C} 4 \mathrm{~b} / \mathrm{c}\left(\mathrm{r}_{\mathrm{p}}{ }^{2}=0.63\right)$, and $\mathrm{C} 6\left(\mathrm{r}_{\mathrm{p}}{ }^{2}=0.81\right)$ was also seen in ex vivo xenoperfusion. Significantly reduced MBL deposition and complement activation was observed on GalTKO/hCD46-PAEC.

Conclusion. Colocalization of MBL/MASP2 with IgM and complement suggests that the lectin pathway is activated by human anti-Gal IgM and may play a pathophysiologic role in pig-to-human xenotransplantation.
\end{abstract}

Keywords: Mannose-binding lectin, Complement pathways, Natural antibody, Xenotransplantation, Porcine aortic endothelial cells.

(Transplantation 2013;96: 791-799)

$\mathrm{T}$ he use of xenografts of pig origin may significantly expand the pool of available donor organs for humans $(1,2)$. However, the major obstacle of pig-to-human xenotransplantation is that grafts are subjected to potent xenorejection processes (3). Hyperacute rejection is the

This study was supported by the Swiss National Science Foundation (32003B_135272, 32003B_138434, and 320030-138376), the Wilsdorf Foundation, and the German Research Council (CRC 127).

The authors declare no conflicts of interest.

${ }^{1}$ Department of Clinical Research, University of Bern, Bern, Switzerland.

2 Graduate School for Cellular and Biomedical Sciences, University of Bern, Bern, Switzerland.

${ }^{3}$ Clinic of Plastic and Hand Surgery, Bern University Hospital, Bern, Switzerland.

${ }^{4}$ Clinic of Cardiovascular Surgery, Bern University Hospital, Bern, Switzerland.

${ }^{5}$ Molecular Animal Breeding and Biotechnology, Gene Center and Department of Veterinary Sciences, LMU Munich, Germany.

${ }^{6}$ Revivicor, Inc., Blacksburg, VA.

7 Division of Immunology and Allergology, University Hospital and Medical Faculty, Geneva, Switzerland.

8 Address correspondence to: Robert Rieben, Ph.D., Department of Clinical Research, University of Bern, Murtenstrasse 50, P.O. Box 44, CH-3010 Bern, Switzerland.

E-mail: robert.rieben@dkf.unibe.ch first and most destructive mechanism $(4,5)$, characterized by rapid loss of graft function after connecting to the host circulation. It is a consequence of the binding of human preformed xenoreactive antibodies against the disaccharide Galo1-3Gal expressed on porcine endothelium (reviewed in Ref. (6)). This antigen-antibody interaction activates the

\footnotetext{
A.K.B. participated in the research design, writing of the article, performance of the research, and data analysis and contributed new reagents or analytic tools. D.K. and H.J. participated in the performance of the animal experiments and the overall design of the study. A.W, A.B., E.W., and N.K. produced GalTKO/hCD46 pigs and provided the respective endothelial cells. D.A. provided primary cells from GalTKO/hCD46 transgenic pigs for nuclear transfer experiments. J.D.S. provided scientific support and reagents and participated in the critical revision of the article. M.A.C. and E.V. participated in the concept and design of the study and carried part of the responsibility. R.R. participated in the concept and design of the study, analysis of the data, and writing of the article and carried the main responsibility for the study.
}

Received 5 June 2013. Revision requested 2 July 2013. Accepted 3 July 2013.

Copyright (C) 2013 by Lippincott Williams \& Wilkins

ISSN: 0041-1337/13/9609-791

DOI: 10.1097/TP.0b013e3182a3a52b 
complement cascade on the endothelial cell membrane, leading to endothelial cell activation and damage, intravascular graft thrombosis, and vessel occlusion (7).

The complement system is activated via three wellcharacterized pathways - the classic, the alternative, and the lectin pathways. Each of them has its own mechanism of activation, resulting in activation of $\mathrm{C} 3$, followed by generation of the membrane attack complex (8). In hyperacute rejection, xenoreactive antibody-mediated activation of the classic pathway is commonly accepted as the main complement activation pathway $(9,10)$. By amplifying the generation of C3b, the alternative pathway also becomes critical in xenograft rejection. However, currently available data do not answer the question whether the lectin pathway may also be involved in the initiation of xenorejection.

The lectin pathway is initiated by interactions between mannose-binding lectin (MBL) and certain carbohydrate arrays (11). MBL naturally exists in a complex with MBLassociated serine proteases (MASPs) $(12,13)$. When MBL binds to a target, the MASPs are activated (14) and cleave their respective substrates- $\mathrm{C} 4$ and C2 for MASP2 and C3 and C2 for MASP1 (15). Historically, the lectin pathway of complement activation is known as antibody-independent pathway. However, several studies using animal models of ischemia-reperfusion injury demonstrated that MBL also interacts with immune complexes to activate complement via the lectin pathway (16-18). In addition, a recent in vitro study suggested that $20 \%$ of human serum IgM could bind to immobilized human MBL $(19,20)$.

In a bypass activation pathway, MBL can directly activate $\mathrm{C} 3$ and the alternative pathway of complement, which are independent of C2 (21). In addition, MBL binding to polymeric IgA induces the initiation of lectin pathway of complement activation (22). Furthermore, myocardial and skeletal ischemia-reperfusion injury studies demonstrated that complement activation and resulting C3 deposition are mediated by $\operatorname{IgM}$ and $\operatorname{MBL}(23,24)$ and independent of $\mathrm{Clq}$ and the classic pathway (24). Taken together, these observations extend the idea that activation of the lectin pathway of complement may not be completely antibody independent.

Thus far, the role of the lectin pathway of complement has not been looked at in xenotransplantation. The present study was therefore focused on lectin pathway activation in pig-to-human xenotransplantation. In particular, we investigated the interaction of MBL and IgM in pig-to-human in vitro as well as ex vivo xenotransplantation models.

\section{RESULTS}

\section{Complement Pathways Involved in Pig-to-Human Xenorejection In Vitro (PAEC Treated with NHS)}

Deposition of Clq (classic pathway), MBL (lectin pathway), and factor $\mathrm{Bb}$ (alternative pathway) on porcine aortic endothelial cells (PAEC) was assessed by cell enzymelinked immunosorbent assay (ELISA). Wild-type (WT) PAEC exposed to 1:2 diluted normal human serum (NHS) showed deposition of IgM, C1q, MBL, factor Bb, C3b/c, C4b/c, and C5b-9 (Fig. 1A). In addition, MBL, C3b/c, and C6 deposition was shown on PAEC treated with 1:10 diluted, MBL-deficient, or Clq-depleted serum or NHS (Fig. 1B). Incubation of WT PAEC with MBL-deficient or C1q-depleted serum also led to expression of adhesion molecules (VCAM-1 and CD62E; Fig. 1C) as well as PAEC cytotoxicity (Fig. 1D).

\section{Lectin Pathway Complement Activation In Vitro-Colocalization Analysis}

Immunofluorescence (IF) staining was performed on WT PAEC treated with 1:10 diluted NHS for the colocalized deposition of MBL with complement proteins. Deposition of MBL on WT PAEC was colocalized with MASP2, C3b/c, $\mathrm{C} 4 \mathrm{~b} / \mathrm{c}$, and C6, suggesting activation of the lectin pathway in this xenotransplantation setting (Fig. 2).

Quantitative colocalization analysis was performed by Manders' correlation coefficient $\left(R_{\mathrm{m}}\right)$ using z-stack confocal images and Imaris software version 7.2.3 (Bitplane). A total overlap of both fluorescent color channels, indicating a perfect three-dimensional colocalization, is indicated by " $R_{\mathrm{m}}=1$," and no colocalization by " $R_{\mathrm{m}}=0$." The Manders' correlation coefficient values were 0.93 for MBL-MASP2, 0.84 for MBL$\mathrm{C} 3 \mathrm{~b} / \mathrm{c}, 0.86$ for MBL-C4b/c, and 0.80 for MBL-C6, suggesting an important contribution of MBL for total complement deposition in this model.

\section{Colocalization of IgM and MBL/MASP2 on WT PAEC}

Colocalization of natural IgM with MBL and MASP2 was investigated on NHS-treated PAEC by confocal microscopy. Colocalized deposition of IgM with MBL and MASP2 was observed on PAEC (yellow staining) (Fig. 3). Manders' colocalization analysis showed near-perfect IgM colocalization with MBL $\left(R_{\mathrm{m}}=0.87\right)$ and MASP2 $\left(R_{\mathrm{m}}=0.83\right)$.

\section{IgM-Dependent MBL and Complement Deposition on WT PAEC}

Binding of MBL to the major porcine xenoantigen Galo1$3 \mathrm{Gal}$ on WT PAEC was assessed in anti-Gal immunoabsorbed and immunoglobulin-depleted (Ig-depleted) serum, respectively. Compared with NHS, reduced MBL deposition was observed on PAEC when incubated with anti-Gal immunoabsorbed NHS or Ig-depleted serum. Purified polyclonal human IgM was used to replete anti-Gal immunoabsorbed NHS and Ig-depleted serum, respectively, which resulted in dose-dependently increased deposition of MBL, C3b/c, and C6 on PAEC (Fig. 3C,D). In addition, WT PAEC were treated with purified human polyclonal IgM followed by incubation with purified MBL protein, resulting in a dosedependently increased binding of purified MBL to PAEC (Fig. 3D).

\section{Complement Regulation by GalTKo/ hCD46 PAEC}

WT or $\alpha$-galactosyl transferase knockout (GalTKO)/ hCD46 PAEC were treated with 1:10 diluted NHS and assessed for complement deposition as well as complementmediated PAEC activation and cytotoxicity. Absence of Gal epitopes and overexpression of hCD46 (Fig. 4) significantly reduced binding of IgM, C1q, MBL, and downstream complement proteins on GalTKO/hCD46 PAEC compared with WT (Fig. 4D). This reduced complement activation on the genetically modified PAEC correlated well with reduced complement-mediated cytotoxicity (Fig. 4E). These observations were also confirmed by IF/confocal microscopy using 
A

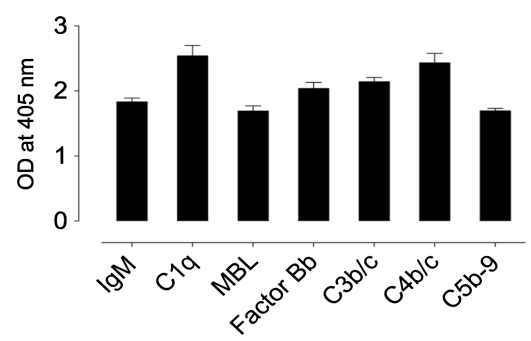

C

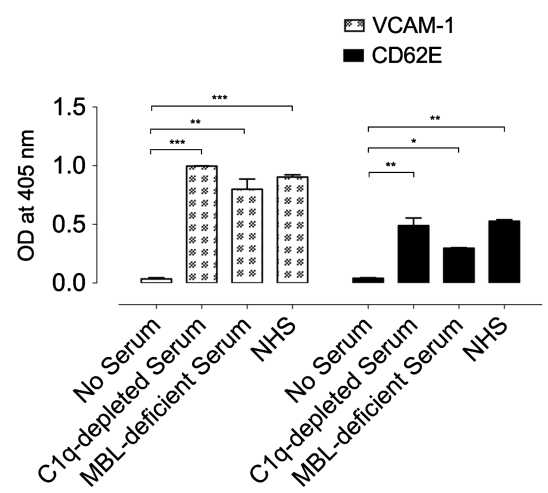

B

Deposition of C1q, MBL, fBb, C3b/c and C6

(61q-depleted serum

$\square$ MBL-deficient serum

- NHS
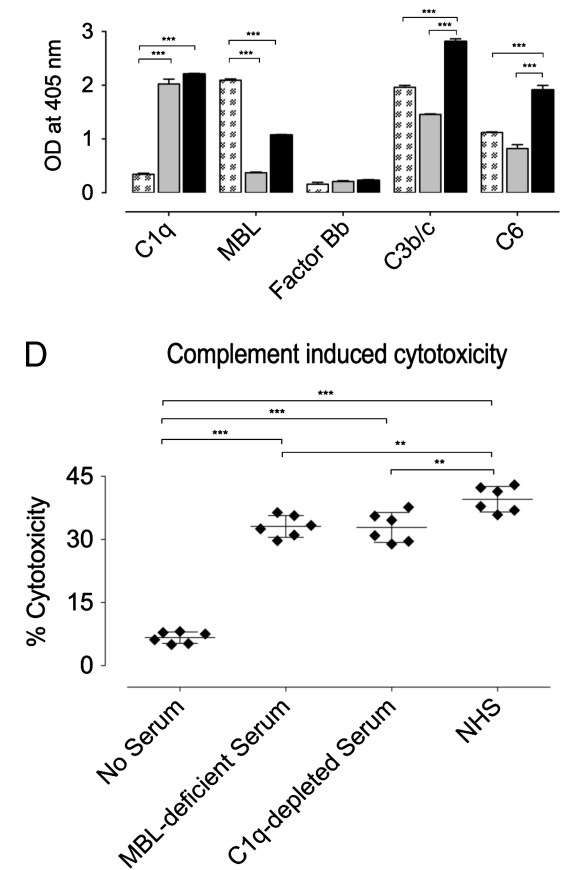

FIGURE 1. Cell ELISA, activation of classic, lectin, and alternative complement pathways in a pig-to-human in vitro xenotransplantation model. A, WT PAEC were treated with $1: 2$ diluted NHS at $37^{\circ} \mathrm{C}$ for 45 min and the deposition of human IgM as well as complement proteins was assessed by specific antibodies. B, treatment of PAEC with 1:10 diluted NHS, MBL-deficient serum and $\mathrm{Clq}$-depleted serum, respectively, and assessment of deposition of $\mathrm{Clq}, \mathrm{MBL}, \mathrm{factor} \mathrm{Bb}, \mathrm{C} 3 \mathrm{~b} / \mathrm{C}$, and $\mathrm{C6}$. C, prolonged incubation ( $4 \mathrm{hr}$ at $37^{\circ} \mathrm{C}$ ) of PAEC with NHS/Clq-depleted serum/MBL-deficient serum (1:10) and assessment of the expression of adhesion molecules VCAM-1 and CD62E. D, treatment of PAEC with 1:10 diluted NHS/Clq-depleted serum/ MBL-deficient serum for $120 \mathrm{~min}$ at $37^{\circ} \mathrm{C}$ and assessment of cytotoxicity by calcein AM and EthD-1. Significance was tested using one-way calcein AM with Bonferroni correction ( ${ }^{*} P<0.05, * * P<0.01$, $\left.* * * P<0.001\right)$. Data are mean \pm SD of three independent experiments.

WT and GalTKO/hCD46 PAEC treated with 1:10 diluted NHS (Fig. 4F).

\section{Analysis of Lectin Pathway Complement Activation} during Ex Vivo Pig Limb Xenoperfusion

Extracorporeal perfusion of WT porcine forelimbs with heparin anticoagulated human blood did not reveal signs of hyperacute rejection and lasted for at least $12 \mathrm{hr}$, after which it had to be terminated due to continuous small blood losses. Blood gas analysis showed constant physiologic potassium, lactate ( $10.78 \pm 0.63)$, and $\mathrm{pH}$ values (7.32 \pm 0.06$)$. All limbs showed full muscular response on neural stimulation throughout the experiments. Stable and physiologic hemodynamic perfusion parameters (pressure, flow) could be maintained throughout all perfusions. To prevent blood loss due to bleeding, biopsy samples were collected only at later time points of perfusion, except for one experiment where samples were collected $1 \mathrm{hr}$ after the onset of perfusion. These biopsy samples were used to analyze deposition of MBL, C1q, and factor $\mathrm{Bb}$ (Fig. 5A-C).

Tissue samples taken after $12 \mathrm{hr}$ perfusion were stained for deposition of human IgM, MBL, C3b/c, C4b/c, and C6. Analysis by fluorescence microscopy revealed that IgM, C3b/c, $\mathrm{C} 4 \mathrm{~b} / \mathrm{c}$, and $\mathrm{C} 6$ were codeposited with human MBL in the tissue. Quantitative two-dimensional colocalization analysis was performed by Pearson's correlation coefficient $\left(R_{\mathrm{p}}\right)$ using fluorescence microscopy images and Imaris software. A total overlap of both fluorescent color channels, represented as " $R_{\mathrm{p}}=+1$," indicates a perfect colocalization, " $R_{\mathrm{p}}=0$ " random colocalization, and " $R_{\mathrm{p}}=-1$ " perfect exclusion. The $R_{\mathrm{p}}$ values were 0.88 for IgM-MBL, 0.82 for $\mathrm{C} 3 \mathrm{~b} / \mathrm{c}-\mathrm{MBL}, 0.63$ for C4b/c-MBL, and 0.81 for C6-MBL (Fig. 5D-S).

\section{DISCUSSION}

The role of complement activation in the pathogenesis of pig-to-human xenograft rejection is well established. However, almost nothing is known to date about the activation of the lectin pathway of complement in xenorejection. We demonstrate here for the first time that all three pathways of complement are activated in a model of pig-to-human xenotransplantation. We could show full activation of complement, including formation of the terminal complement complex and complement-mediated cytotoxicity also in the absence of C1q. In addition, our data indicate that activation of the MBL route of complement is dependent on IgM, both in vitro and ex vivo, in a pig-to-human xenotransplantation model. 

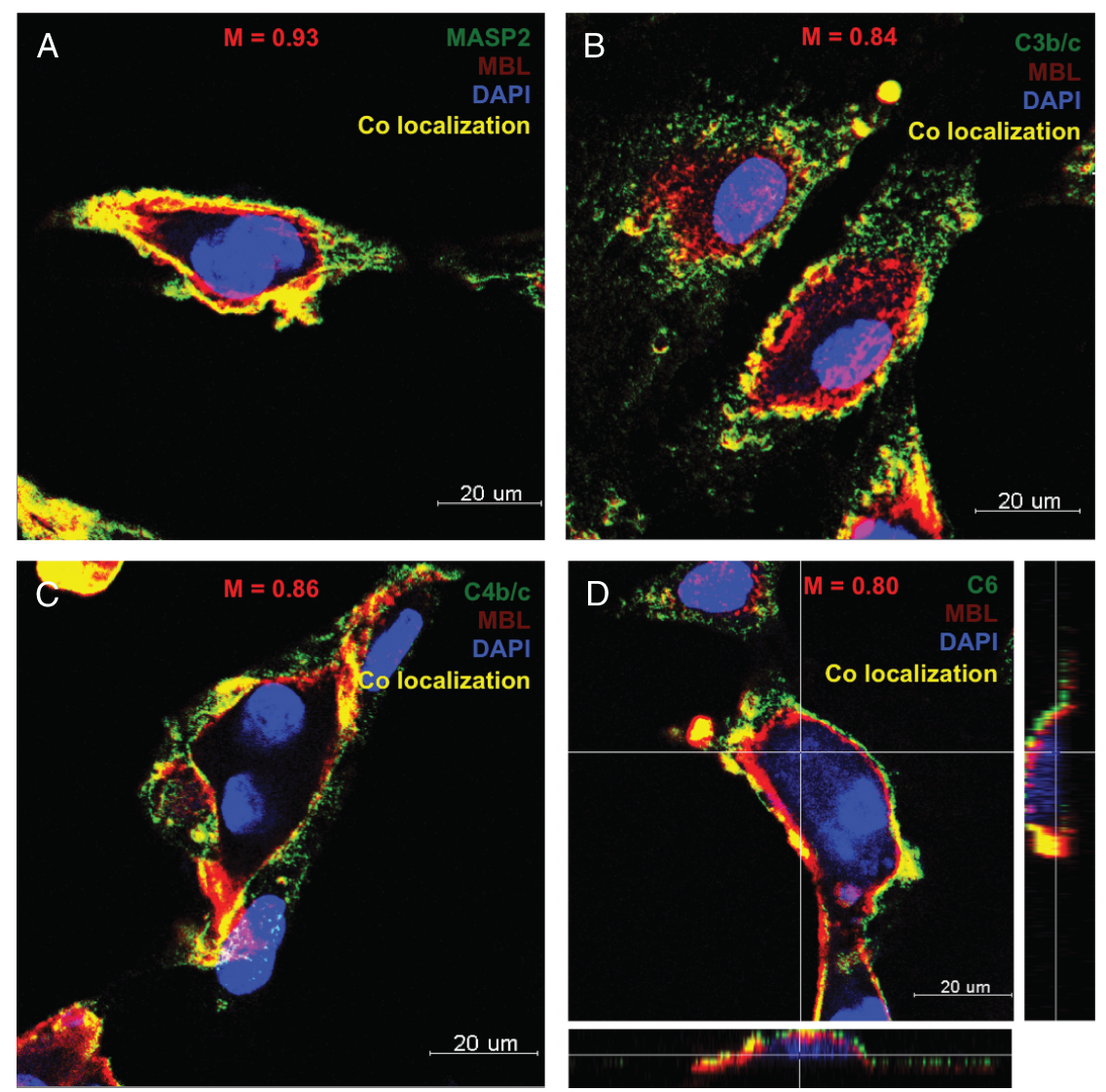

FIGURE 2. Colocalization analysis of MBL with other complement proteins. NHS (1:10) treated WT PAEC were stained for the deposition of MBL (red fluorescence) and MASP2, C3b/c, C4b/c, and C6, respectively (green fluorescence). Representative colocalization images of MBL with the respective Manders' correlation coefficients are shown: $(\mathrm{A}) \mathrm{MASP} 2\left(R_{\mathrm{m}}=0.93\right),(\mathrm{B}) \mathrm{C} 3 \mathrm{~b} / \mathrm{c}$ $\left(R_{\mathrm{m}}=0.84\right)$, (C) C4b/c $\left(R_{\mathrm{m}}=0.86\right)$, and (D) C6 $\left(R_{\mathrm{m}}=0.80\right)$. Colocalization was quantified by z-series analysis using Manders' correlation coefficient. For MBL-C6 colocalization (D), also side views are shown as z-panels to the right and below the image.

Endothelium is the first cell type to make contact with the recipients' blood in xenotransplantation or xenoperfusion. We therefore chose to analyze the effects of human serum on PAEC in vitro to perfuse porcine limbs ex vivo with whole, heparin-anticoagulated human blood. PAEC treated with NHS showed deposition of MBL, C1q, and factor $\mathrm{Bb}$ on their surface, indicating that all three pathways are involved in complement-mediated xenograft damage. In addition, deposition of $\mathrm{C} 4 \mathrm{~b} / \mathrm{c}, \mathrm{C} 3 \mathrm{~b} / \mathrm{c}, \mathrm{C} 6$, and $\mathrm{C} 5 \mathrm{~b}-9$, detected as $\mathrm{C} 9$ neoepitope, were also observed on NHS-treated PAEC. The same markers, indicating full activation of complement, were also found when C1q-depleted serum was used. Furthermore, C1q-depleted serum induced PAEC activation, as measured by expression of the adhesion molecules E-selectin and VCAM-1, as well as PAEC cytotoxicity in a complement-dependent manner.

Binding of MBL was colocalized with C3b/c, C4b/c, and C6 on PAEC, suggesting that deposition of MBL on the cells plays a functional role in complement activation. Because natural antibodies are the major contributors to complement activation, the involvement of IgM in lectin pathway activation was then investigated. Colocalization studies on PAEC treated with NHS revealed that MBL/MASP2 deposition was colocalized with IgM, quantitative Manders' coefficient values indicating a near $100 \%$ colocalization, suggesting that IgM and MBL may work together in a synergetic way. The importance of anti-Gal IgM in lectin pathway activation was further confirmed by depletion of anti-Gal antibodies from NHS by immunoabsorption, which led to a significantly reduced binding of IgM as well as MBL on PAEC and also to a reduction of C5b-9 deposition. Repletion of immunoabsorbed or total Ig-depleted serum with polyclonal human IgM resulted in a dose-dependently increased deposition of IgM, MBL, and further downstream complement components. In addition, a dose-dependent deposition of purified MBL was found on PAEC treated with human polyclonal IgM. Taken together, these data indicate that the activation of the lectin pathway of complement in this xenotransplantation model is dependent on IgM binding to the target cells. This finding is in line with a recent study showing MBL binding sites on IgM, containing mainly GlcNActerminated glycans, which are strong ligands for MBL (20). Also, the oligomannose structures present on IgM at Asn-402 and Asn-563 could provide binding sites for $\operatorname{MBL}(25,26)$.

Knockout of the Gal epitope in pigs is currently being combined with overexpression of human complement regulatory proteins such as hCD46 to prevent pig-to-human xenograft rejection. In this study, PAEC from GalTKO/hCD46 

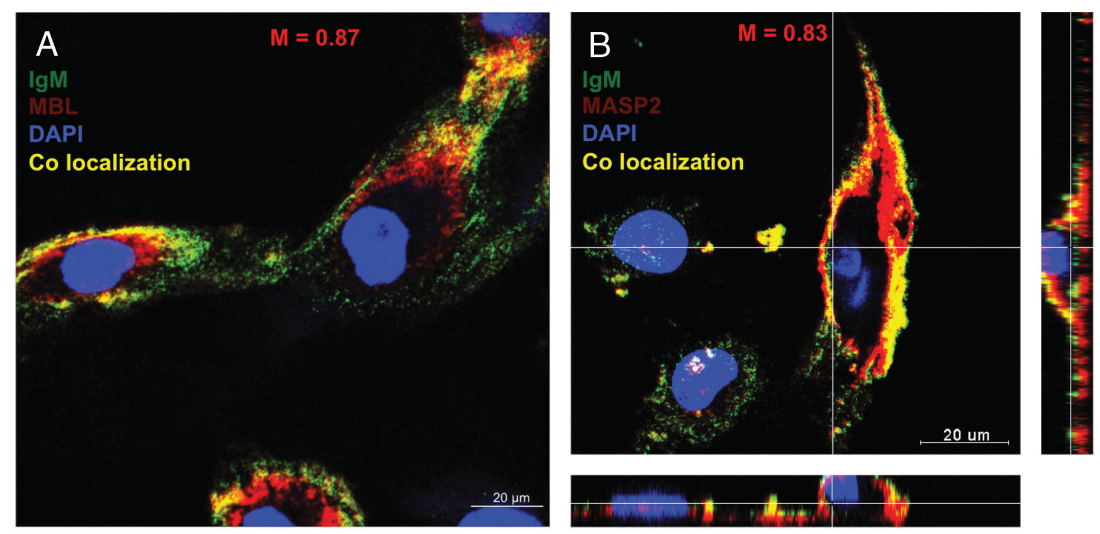

C Deposition of IgM, C1q, MBL, C3b/c and C6 on PAEC

D Importance of (anti-Gal) IgM for binding of MBL to PAEC
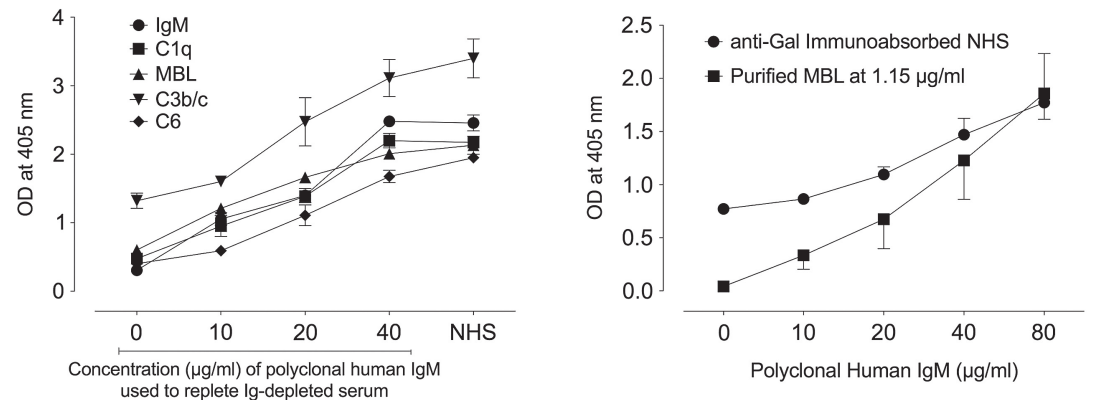

FIGURE 3. Role of IgM in the activation of lectin pathway. Colocalization (yellow) of IgM (green) with MBL and MASP2 (red), respectively. Representative colocalization images of IgM with the respective Manders' correlation coefficients are shown: (A) IgM-MBL $\left(R_{\mathrm{m}}=0.87\right)$ and (B) IgM-MASP2 $\left(R_{\mathrm{m}}=0.83\right)$ on NHS-treated WT PAEC. For IgM-MASP2 colocalization (B), also side views are shown as z-panels to the right and below the image. IgM-dependent MBL and complement deposition on PAEC. Deposition of MBL and complement components on PAEC was tested in the absence (anti-Gal immunoabsorbed NHS/Ig-depleted serum) or presence of anti-Gal IgM (repletion with polyclonal human IgM). C, deposition of IgM, Clq, $\mathrm{MBL}, \mathrm{C} 3 \mathrm{~b} / \mathrm{c}$, and $\mathrm{C} 6$ on PAEC incubated with Ig-depleted serum repleted with increasing concentrations of purified human IgM as well as NHS. D, binding of MBL to PAEC in the presence anti-Gal immunoabsorbed NHS (1:10) or purified MBL at a fixed concentration ( $1.15 \mu \mathrm{g} / \mathrm{mL})$. MBL-binding curves for addition of increasing concentrations of purified human IgM are shown. Data are mean \pm SD of three independent experiments.

pigs showed significantly reduced deposition of MBL and complement proteins as well as no cytotoxicity when treated with NHS. These observations suggest that GalTKO and expression hCD46 could also help to prevent lectin pathway activation during pig-to-human xenotransplantation.

Tissue samples from porcine forelimbs ex vivo xenoperfused with whole, heparin-anticoagulated human blood were analyzed for the involvement of IgM-mediated lectin pathway activation. Deposition of $\mathrm{MBL}, \mathrm{Clq}$, and factor $\mathrm{Bb}$ was observed on $1 \mathrm{hr}$ perfusion samples, suggesting that all three pathways of complement are activated. In addition, MBL was colocalized with IgM in $12 \mathrm{hr}$ perfusion samples and the finding of colocalized deposition of MBL with C3, C4, and C6 indicates that also in ex vivo xenoperfusion the MBL route of complement is functional, confirming the in vitro data.

In conclusion, our results demonstrate activation and pathogenic role of the lectin pathway of complement in xenotransplantation. Colocalized deposition of $\operatorname{IgM}$ with MBL and MASP2 indicates that activation of the lectin pathway of complement in this pig-to-human xenotransplantation model is dependent on IgM.

\section{MATERIALS AND METHODS}

\section{Cell ELISA and Determination of Complement Activation Pathways in Xenotransplantation}

A modified whole-cell ELISA was used to detect the complement pathways involved in xenotransplantation, similar to methods described previously $(27,28)$. WT PAEC as well as GalTKO/hCD46 transgenic PAEC were grown to confluence in 96-well plates and washed twice with ELISA wash buffer supplemented with or without calcium $(45 \mathrm{mM} \mathrm{NaCl}, 10 \mathrm{mM}$ HEPES, $680 \mathrm{mM} \mathrm{CaCl}_{2}$, and $490 \mathrm{mM} \mathrm{MgCl}_{2}$ ). Pooled NHS or alternatively C1qdepleted serum (A509; Quidel, Darmstadt, Germany) or MBL-deficient serum (HSR002; Statens Serum Institut, Copenhagen, Denmark) in $\mathrm{GVB}^{++}$ ( $1: 2$ or $1: 10$ ) was added to the cells and incubated at $37^{\circ} \mathrm{C}$ followed by washing with ELISA wash buffer. Cells were fixed in $1 \%$ paraformaldehyde for $15 \mathrm{~min}$ at room temperature (RT), washed, and blocked with phosphate-buffered saline (PBS)/1\% bovine serum albumin (BSA) for $90 \mathrm{~min}$ at RT. Goat antihuman IgM (fluorescein isothiocyanate [FITC] labeled; catalog number F5384; Dako, Carpinteria, CA), rabbit anti-human Clq (A0136; Dako), mouse anti-human MBL (clone 15C5; sc80598; Santa Cruz Biotechnology, Santa Cruz, CA), rabbit anti-human factor Bb (ab72658; Abcam, Cambridge, UK), rabbit anti-human C3b/c (FITC labeled; A0062; Dako), rabbit anti-human C4b/c (FITC labeled; F0169; Dako), mouse anti-human C5b-9 (aE11; 5010; Diatec, Oslo, Norway), mouse anti-human CD62E (LS-C13922; LifeSpan, 
A
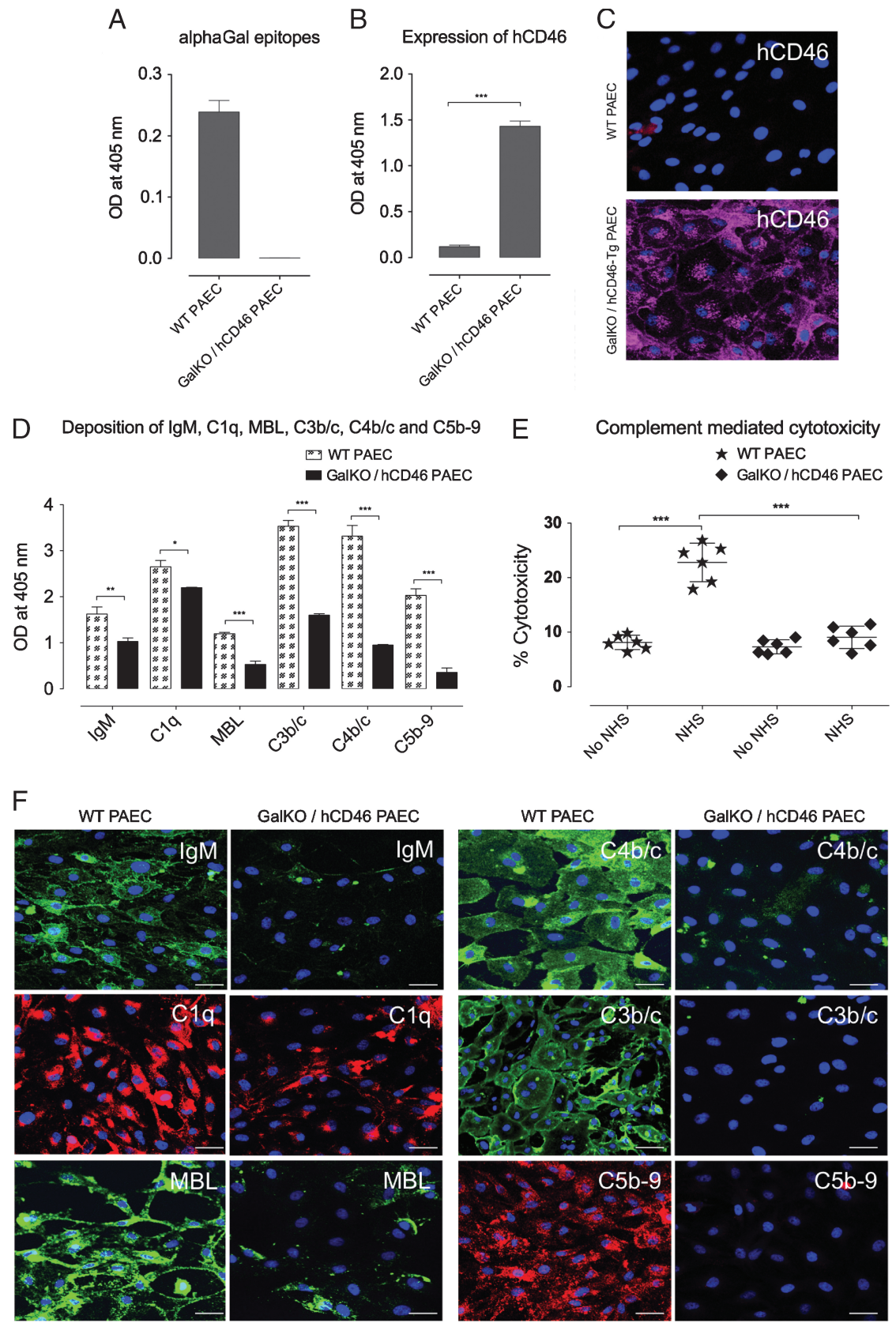

GalKO / hCD46 PAEC
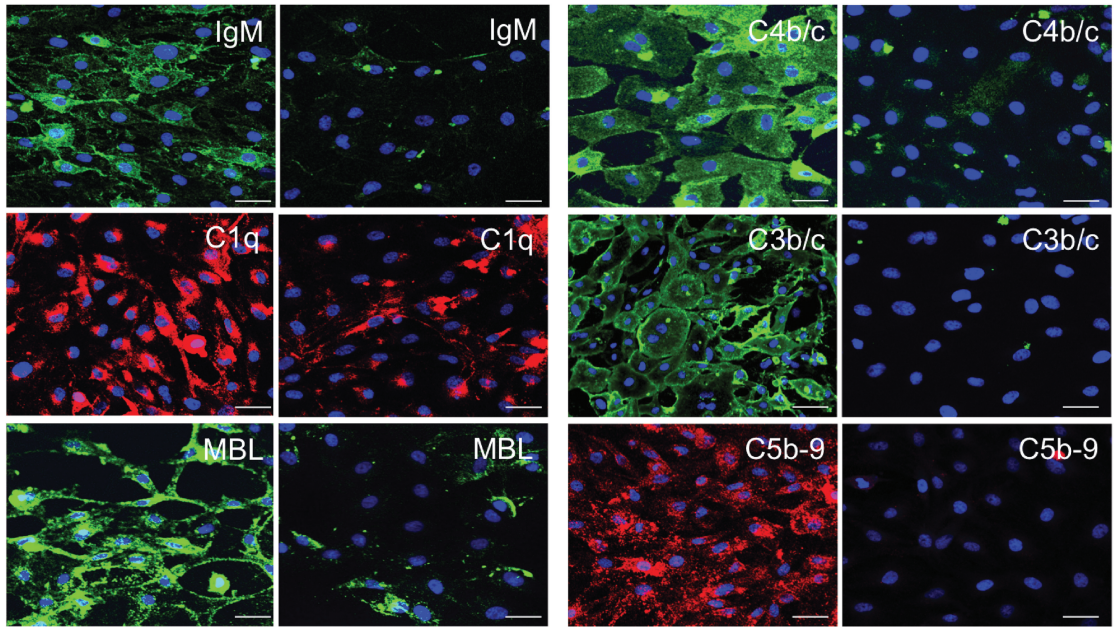

FIGURE 4. Regulation of complement activation on PAEC using genetic modification strategies. (A) Gal epitope and (B and C) hCD46 expression on WT and GalTKO/hCD46 PAEC analyzed by cell ELISA and IF staining. D, deposition of IgM, Clq, MBL, C3b/c, C4b/c, and C5b-9 on NHS (1:10)-treated WT and GalTKO/hCD46 PAEC. E, treatment of PAEC with l:10 diluted NHS for $120 \mathrm{~min}$ at $37^{\circ} \mathrm{C}$ and assessment of cytotoxicity by calcein AM and EthD-l. F, confocal analysis of IgM, Clq, MBL, C4b/c, C3b/c, and C5b-9 on WT and GalTKO/hCD46 PAEC treated with 1:10 diluted NHS. Significance was tested using one-way analysis of variance with Bonferroni correction $(* P<0.05, * * P<0.01, * * * P<0.001)$. Data are mean \pm SD of three independent experiments. Scale bars, $30 \mu \mathrm{m}$.

Seattle, WA), mouse anti-human VCAM-1 (1G11B1; 9510; Southern Biotech, Birmingham, AL), mouse anti- $\alpha$ Gal (4F10; a gift of A. Bendelac, Howard Hughes Medical Institute [Chevy Chase, MD] and University of Chicago [Chicago, IL]), and mouse anti-human CD46 (HM2103; Hycult Biotech, Plymouth Meeting, PA) were diluted in PBS/1\% BSA and incubated for $1 \mathrm{hr}$ at RT followed by three washes. Subsequently, biotin-conjugated goat anti-mouse IgG (ab6788; Abcam) or goat anti-rabbit Ig (E0432; Dako) were used, diluted 1:500 in PBS/1\% BSA, and incubated for $1 \mathrm{hr}$ at RT. After washing, alkaline phosphatase-conjugated rabbit anti-FITC (ab49368; Abcam) or streptavidin-alkaline phosphatase (RPN1234V; GE Healthcare,
Cleveland, $\mathrm{OH}$ ) diluted 1:1000 in PBS/1\% BSA was incubated for $30 \mathrm{~min}$ at RT. After washing, $1 \mathrm{mg} / \mathrm{mL}$ p-nitrophenyl phosphate substrate (Sigma, St. Louis, MO) was added. Color development was quantified at $405 \mathrm{~nm}$ (reference $490 \mathrm{~nm}$; Infinite M1000 microplate reader; Tecan, Männedorf, Switzerland).

\section{Complement-Mediated PAEC cytotoxicity}

Complement-induced PAEC cytotoxicity was measured by calcein AM/ ethidium homodimer-1 (EthD-1) staining. A Live/Dead Kit (Molecular Probes, Eugene, OR) was used and protocols provided by the manufacturer 

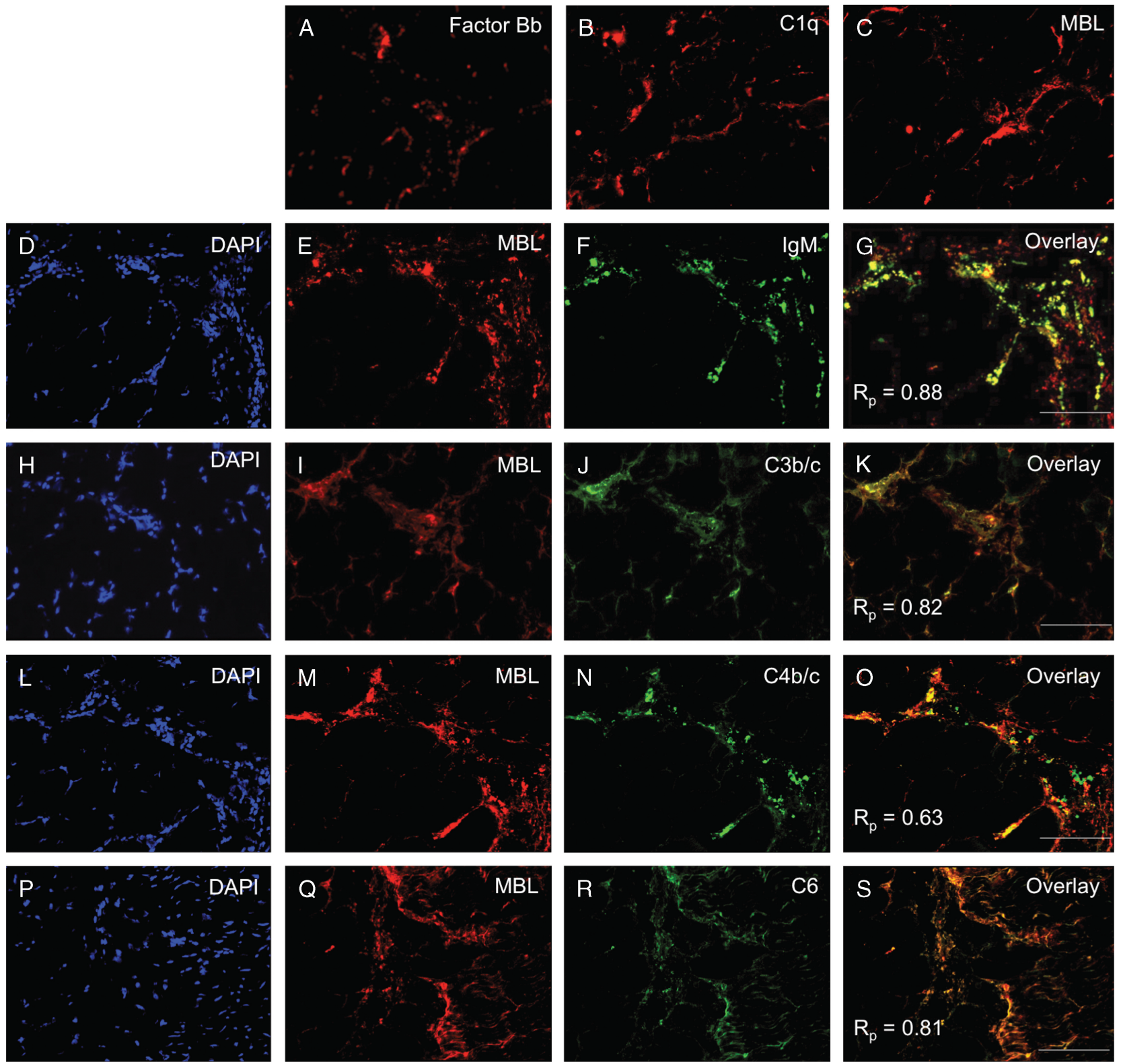

FIGURE 5. Colocalization of MBL with IgM and complement in an ex vivo xenoperfusion model. Tissue samples from ex vivo xenoperfused pig limbs with heparinized, whole human blood for $1 \mathrm{hr}$ were assessed for deposition of (A) MBL, (B) $\mathrm{Clq}$, and (C) factor $\mathrm{Bb}$. Biopsy samples of $12 \mathrm{hr}$ perfusion were analyzed for IgM-mediated lectin pathway activation by using immunofluorescence staining. D-G, deposition of MBL (red), IgM (green), and codeposition (yellow). H-K, deposition of MBL (red), C3b/c (green), and codeposition (yellow). L-O, Deposition of MBL (red), C4b/c (green), and codeposition (yellow). P-S, deposition of MBL (red), C6 (green), and codeposition (yellow). D, H, L, and P, 4',6-diamidino-2-phenylindole staining of nuclei. The $R_{\mathrm{p}}$ values represent Pearson's colocalization coefficient. Representative images of six independent experiments are shown. Scale bars, $100 \mu \mathrm{m}$.

were adopted. Briefly, confluent WT and GalTKO/hCD46 PAEC in a 96-well plate were exposed to 1:10 diluted NHS, C1q-depleted or MBL-deficient serum, for $120 \mathrm{~min}$ at $37^{\circ} \mathrm{C}$. After washing with PBS, $100 \mu \mathrm{L}$ of $1 \mu \mathrm{M}$ calcein $\mathrm{AM}$ and $2 \mu \mathrm{M}$ EthD-1 was added to the cells and incubated in dark for $15 \mathrm{~min}$. The fluorescence excitation and emissions of calcein AM and EthD-1 were acquired at 485/535 and 530/635 nm, respectively, using the Infinite M1000 microplate reader (Tecan).

\section{IF Staining}

Activation of the lectin pathway of complement was further investigated by immunofluorescence staining using confocal microscopy. Briefly, WT and GalTKO/hCD46 PAEC grown to confluence on eight-well Lab-Tek chamber slides (Milian) were washed twice with $\mathrm{PBS}^{++}$(PBS containing $1 \mathrm{mM} \mathrm{CaCl}_{2}$ and $1 \mathrm{mM} \mathrm{MgCl}_{2}$ ) and treated with 1:10 dilution NHS for 45 to $60 \mathrm{~min}$ at RT. Slides were washed thrice and fixed with parapicric acid for 15 min at RT. After washing and blocking with PBS/3\% BSA for 30 min at RT, slides were incubated for $60 \mathrm{~min}$ with primary antibodies for the following antigens: IgM (FITC labeled; F5380; Sigma), MBL (15C5; Santa Cruz Biotechnology), MASP2 (sc-17905; Santa Cruz Biotechnology), C4b/c-FITC (Dako), C3b/c-FITC (Dako), and C6 (A307; Quidel) diluted in PBS/1\% BSA. After washing, fluorescence-labeled specific secondary antibodies diluted in 
PBS/1\% BSA were incubated for $60 \mathrm{~min}$ and the slides were mounted with glycergel (C0563; Dako). The stained slides were then analyzed using a Zeiss LSM5 confocal laser scanning microscope.

\section{Immunoabsorption of Human Serum on PAA-Bdi Sepharose}

To investigate the importance of anti-Gal $\alpha 1-3 \mathrm{Gal}$ IgM for MBL binding to PAEC, immunoabsorption of NHS was carried out to eliminate anti-Gal antibodies from NHS. Conjugates of the B-disaccharide Gal 1 -3Gal (Bdi) and poly- $N$-hydroxyethylacrylamide (PAA-Bdi), covalently linked to Sepharose $6 \mathrm{FF}$ (PAA-Bdi Sepharose), were obtained from Dr. Nicolai Bovin (Moscow, Russia). Mini-spin columns were packed with 100 or $200 \mu \mathrm{L}$ PAA-Bdi Sepharose and rinsed with PBS (10-15 min, $\left.200 \mathrm{rpm}, 0^{\circ} \mathrm{C}\right)$. NHS $(450 \mu \mathrm{L})$ was absorbed over the columns. Thereafter, immunoabsorbed NHS aliquots were prepared and stored at $-80^{\circ} \mathrm{C}$ until use.

\section{Role of IgIM on MBL Binding to PAEC}

The influence of IgM on binding of MBL to PAEC was investigated by cell ELISA using immunoabsorbed NHS and Ig-depleted serum (SunnyLab, Kent, UK). Human polyclonal IgM (A50168H; Meridian Life Science, Cincinnati, $\mathrm{OH})$ was used to replete immunoabsorbed NHS/Ig-depleted serum. Binding of purified MBL (HSR008; Statens Serum Institut) on PAEC in the presence of human polyclonal IgM was also tested.

\section{Ex vivo Xenoperfusion of Pig Limbs with Whole, Anticoagulated Human Blood}

Six forelimbs of WT pigs were used to perfuse with whole, heparinanticoagulated human blood $(29,30)$. Large white pigs were premedicated and anesthetized with ketamine, xylazine, midazolam, and atropine and mechanically ventilated $\left(\mathrm{O}_{2} /\right.$ air $1: 3$, isoflurane $1-1.5$ vol.\%). Forelimbs were amputated by dissection of the shoulder girdle muscles using an electrocautery device (ICC 350; ERBE Elektromedizin, Tübingen, Germany). The neurovascular bundle in the axillar region was laid open and two veins and one artery of the amputated forelimb were cannulated using 10 French cannulas. All animal experiments were conducted in compliance with the Guide for the Care and Use of Laboratory Animals (NIH Publication No. 85-23, revised 1996) and Swiss national guidelines. The study was approved by the local animal experimentation committee of the Canton of Bern.

Five hundred milliliters of blood each were withdrawn from individual human donors into standard transfusion bags to which 10,000 IU heparin were added. The porcine forelimbs were attached to extracorporeal perfusion circuits as described previously (29). Xenogeneic perfusion of the porcine limbs was then performed with the whole, heparin-anticoagulated human blood. The perfusion parameters (arterial blood gas, response to nerve stimulation, etc.) were monitored throughout the experiment.

Skeletal muscle biopsies were collected before perfusion from the contralateral extremity as baseline samples. Different time-point biopsies were collected after $1 \mathrm{hr}(\mathrm{n}=1)$ and $12 \mathrm{hr}(\mathrm{n}=6)$ of perfusions. Samples were fixed in $2 \%$ buffered formaldehyde solution for $24 \mathrm{hr}$ and then transferred into $18 \%$ sucrose for $15 \mathrm{hr}$. Then, the samples were embedded in Shandon M1 embedding matrix (Thermo Scientific, Waltham, MA) and stored at $-20^{\circ} \mathrm{C}$ until sectioned.

\section{IF Staining for IgM-Dependent Lectin Pathway Activation on Ex Vivo Xenoperfusion Samples}

IF staining was performed on ex vivo xenoperfusion biopsy samples for the deposition of MBL and its colocalization with IgM, C3b/c, C4b/c, and C6. In brief, $20-\mu \mathrm{m}$-thick sections were cut, fixed on slide, and treated with TBSTriton X-100 for 15 min. Deposition of MBL and its colocalization with complement proteins was assessed by using specific antibodies as mentioned above. Stained slides were observed using fluorescence microscopy (DMI 4000B; Leica, Wetzlar, Germany).

\section{ACINOWLEDGIMENTS}

The authors thank Prof. Nicolai Bovin and Dr. Elena Korchagina (Moscow, Russia) for the kind gift of PAA-Bdi
Sepharose for immunoabsorption of anti-Gal antibodies. Expert technical support was provided by Dr. Daniel Mettler, Mrs. Olgica Beslac, and Mr. Daniel Zalokar (Experimental Surgery Laboratory, Department of Clinical Research, University of Bern).

\section{REFERENCES}

1. Yang YG, Sykes M. Xenotransplantation: current status and a perspective on the future. Nat Rev Immunol 2007; 7: 519.

2. Ekser B, Ezzelarab M, Hara H, et al. Clinical xenotransplantation: the next medical revolution? Lancet 2012; 379: 672.

3. Platt JL, Bach FH. The barrier to xenotransplantation. Transplantation 1991; 52: 937.

4. Lawson JH, Platt JL. Molecular barriers to xenotransplantation. Transplantation 1996; 62: 303.

5. Dalmasso AP, Vercellotti GM, Fischel RJ, et al. Mechanism of complement activation in the hyperacute rejection of porcine organs transplanted into primate recipients. Am J Pathol 1992; 140: 1157.

6. Galili U. Interaction of the natural anti-Gal antibody with alphagalactosyl epitopes: a major obstacle for xenotransplantation in humans. Immunol Today 1993; 14: 480.

7. Platt JL, Fischel RJ, Matas AJ, et al. Immunopathology of hyperacute xenograft rejection in a swine-to-primate model. Transplantation 1991; 52: 214.

8. Walport MJ. Complement. First of two parts. N Engl J Med 2001; 344: 1058 .

9. Roos A, Daha MR. Antibody-mediated activation of the classical complement pathway in xenograft rejection. Transpl Immunol 2002; 9: 257.

10. Walpen AJ, Mohacsi P, Frey C, et al. Activation of complement pathways in xenotransplantation: an in vitro study. Transpl Immunol 2002; 9: 271.

11. Turner MW. Mannose-binding lectin: the pluripotent molecule of the innate immune system. Immunol Today 1996; 17: 532.

12. Thiel S, Vorup-Jensen T, Stover CM, et al. A second serine protease associated with mannan-binding lectin that activates complement. Nature 1997; 386: 506.

13. Turner MW. The lectin pathway of complement activation. Res Immunol 1996; 147: 110

14. Vorup-Jensen T, Petersen SV, Hansen AG, et al. Distinct pathways of mannan-binding lectin (MBL)- and C1-complex autoactivation revealed by reconstitution of $\mathrm{MBL}$ with recombinant $\mathrm{MBL}$-associated serine protease-2. J Immunol 2000; 165: 2093.

15. Hajela K, Kojima M, Ambrus G, et al. The biological functions of MBL-associated serine proteases (MASPs). Immunobiology 2002; 205: 467.

16. Zhang M, Takahashi K, Alicot EM, et al. Activation of the lectin pathway by natural IgM in a model of ischemia/reperfusion injury. $J$ Immunol 2006; 177: 4727.

17. McMullen ME, Hart ML, Walsh MC, et al. Mannose-binding lectin binds IgM to activate the lectin complement pathway in vitro and in vivo. Immunobiology 2006; 211: 759.

18. Busche MN, Pavlov V, Takahashi K, et al. Myocardial ischemia and reperfusion injury is dependent on both $\operatorname{IgM}$ and mannose-binding lectin. Am J Physiol Heart Circ Physiol 2009; 297: H1853.

19. Nevens JR, Mallia AK, Wendt MW, et al. Affinity chromatographic purification of immunoglobulin $\mathrm{M}$ antibodies utilizing immobilized mannan binding protein. J Chromatogr 1992; 597: 247.

20. Arnold JN, Wormald MR, Suter DM, et al. Human serum IgM glycosylation: identification of glycoforms that can bind to mannanbinding lectin. J Biol Chem 2005; 280: 29080.

21. Selander B, Mårtensson U, Weintraub A, et al. Mannan-binding lectin activates C3 and the alternative complement pathway without involvement of C2. J Clin Invest 2006; 116: 1425.

22. Roos A, Bouwman LH, van Gijlswijk-Janssen DJ, et al. Human IgA activates the complement system via the mannan-binding lectin pathway. J Immunol 2001; 167: 2861.

23. Jordan JE, Montalto MC, Stahl GL. Inhibition of mannose-binding lectin reduces postischemic myocardial reperfusion injury. Circulation 2001; 104: 1413.

24. Walsh MC, Bourcier T, Takahashi K, et al. Mannose-binding lectin is a regulator of inflammation that accompanies myocardial ischemia and reperfusion injury. J Immunol 2005; 175: 541. 
25. Chapman A, Kornfeld R. Structure of the high mannose oligosaccharides of a human IgM myeloma protein. I. The major oligosaccharides of the two high mannose glycopeptides. J Biol Chem 1979; 254: 816.

26. Chapman A, Kornfeld R. Structure of the high mannose oligosaccharides of a human IgM myeloma protein. II. The minor oligosaccharides of high mannose glycopeptide. J Biol Chem 1979; 254: 824.

27. Haraldsen G, Kvale D, Lien B, et al. Cytokine-regulated expression of E-selectin, intercellular adhesion molecule-1 (ICAM-1), and vascular cell adhesion molecule-1 (VCAM-1) in human microvascular endothelial cells. J Immunol 1996; 156: 2558.

28. Platt JL, Turman MA, Noreen HJ, et al. An ELISA assay for xenoreactive natural antibodies. Transplantation 1990; 49: 1000.

29. Constantinescu MA, Knall E, Xu X, et al. Preservation of amputated extremities by extracorporeal blood perfusion; a feasibility study in a porcine model. J Surg Res 2011; 171: 291.

30. Muller S, Constantinescu MA, Kiermeir DM, et al. Ischemia/reperfusion injury of porcine limbs after extracorporeal perfusion. J Surg Res 2013; 181: 170. 